\title{
Efectos de la Asimetría de Género en la Representación Social del Desempleo Femenino
}

\section{The Effects of Gender Asymmetry in the Social Representation of Female Unemployment}

\author{
Maider Larrañaga, José F. Valencia y Garbiñe Ortiz \\ Universidad del País Vasco/Euskal Herriko Unibertsitatea
}

\begin{abstract}
Basándose en la teoría del núcleo central (Abric, 1976, 1987), el objetivo del estudio fue analizar la representación social que jóvenes en formación profesional tienen sobre el desempleo masculino y femenino. En el estudio participaron 231 jóvenes de 3 centros de formación tecnológica elegidos intencionadamente del Departamento de los Pirineos Atlánticos en Francia, los que respondieron a un cuestionario con preguntas de evocación y de refutación. Los resultados del análisis lexicográfico y categorial mostraron las especificidades del desempleo femenino, organizado en relación a la dimensión familiar, y las del masculino, organizado en relación a la dimensión individual. Los porcentajes de refutación, por su parte, mostraron que los elementos centrales del desempleo femenino y del masculino son los mismos. Estos resultados evidencian la organización en base al género de la representación social del desempleo, en la que sigue vigente la asignación tradicional de los roles de género.
\end{abstract}

Palabras clave: desempleo, asimetría de género, representaciones sociales, núcleo central, sistema periférico

\begin{abstract}
Based on the theory of the central core (Abric, 1976, 1987), the aim of the study was to analyze the social representation of male and female unemployment held by young people with vocational training studies. In the study 231 students from 3 purposively chosen technology training centers in the Pyrénées-Atlantiques Department in France completed a questionnaire containing evocation and refutation items. The results of the lexicographic and categorical analysis showed the specificities of female unemployment, organized along the family dimension and of male unemployment, organized around the individual dimension. In contrast, percentages related to the refutation questionnaires showed the same core elements in both female and male unemployment. These results suggest that the social representation of unemployment is differently organized as a function of gender, thus implying that the traditional views of gender roles still persist.
\end{abstract} system

Keywords: unemployment, gender asymmetry, social representation, central core, peripheral

La forma en la que se analiza y presenta la problemática del desempleo responde a determinados intereses. El desempleo se presenta como un fenómeno general, pero, evidentemente, afecta a la población de forma desigual. Concretamente, el desempleo femenino, más allá de algunas manifestaciones y acciones políticas, sigue siendo considerado como una problemática secundaria. Las mujeres que se encuentran en situación de desempleo a menudo son clasificadas como inactivas, independientemente de si el motivo es por falta de perspectiva de inserción laboral o para dedicarse al cuidado de los hijos (Aquilini \& Costa, 2003), asignándoles el rol tradicional de amas de casa (Valencia \& Larrañaga, 2004). A la hora de explicar el sobre-desempleo femenino, Torns (1998/2000) señala que la sociedad percibe a las mujeres jóvenes como futuras madres y esposas y no como desempleadas. Al parecer, las mujeres son especialmente víctimas de la de-

Maider Larrañaga, José F. Valencia y Garbiñe Ortiz, Departamento de Psicología Social y Metodología de las Ciencias del Comportamiento, Universidad del País Vasco/Euskal Herriko Unibertsitatea, Donostia, España.

Los autores agradecen al proyecto SEJ2007-67193/PSIC la posibilidad de la realización de este trabajo.

La correspondencia relativa a este artículo debe ser dirigida a Maider Larrañaga, Departamento de Psicología Social y Metodología de las Ciencias del Comportamiento, Facultad de Psicología, Universidad del País Vasco, Avda. de Tolosa, 70, 20018 Donostia, España. E-mail: maider.larranaga@ehu.es 
gradación general de las condiciones de empleo y de un empobrecimiento tolerado (Cozzarelli, Tagler \& Wilkinson, 2002).

En las últimas décadas, junto con el reconocimiento del trabajo de las mujeres, asistimos a una incorporación masiva de las mujeres al trabajo asalariado como reivindicación de igualdad. La Organización Internacional del Trabajo (OIT) señala la tendencia general del aumento de la participación de las mujeres en los mercados de trabajo del mundo, pero con una sobrerrepresentación de la precariedad laboral y el desempleo (OIT, 2007). El mismo informe señala que las tasas de desempleo juvenil son más elevadas que las de los adultos y que la dificultad para encontrar trabajo es mayor para las mujeres entre 15 y 24 años. Duru-Bellat (1990) evidencia la paradoja que sufren las jóvenes, ya que normalmente obtienen buenos resultados académicos, pero sufren más dificultades en su inserción profesional. Para explicar estas desigualdades, Vouillot, Blanchard, Maro y Steinbruckner (2004) ponen el acento en la división sexual de la orientación escolar y del trabajo.

En el estudio que reportamos en este artículo pretendimos replicar, con otra población de estudiantes, el estudio previo realizado por Valencia y Larrañaga (2004) con estudiantes de formación profesional del departamento de Provenza Alpes-Costa Azul (Francia), en el que se evidenció la influencia de la división de roles entre hombres y mujeres en la representación social del desempleo. Nuestro interés con este nuevo estudio fue aumentar la generalización de los resultados y dar cuenta de los mecanismos que regulan y perpetúan la asimetría de género. Para ello, quisimos profundizar en los mecanismos ideológicos que legitiman la representación, articulando el concepto de asimetría de género con la aproximación estructural de las representaciones sociales (RS). También quisimos analizar la influencia de la pertenencia de género en la representación social del desempleo y en sus concreciones (desempleo masculino y femenino).

\section{La Asimetría de Género}

En la teoría del campo social desarrollada por Bourdieu $(1977,1998)$ el espacio social se encuentra organizado por posiciones sociales determinadas en función de tres dimensiones: el capital económico, el capital cultural y el género. Los individuos están inmersos en posicionamientos asimétricos diferentes, en los que se reflejan las luchas, retos y relaciones de poder que estructuran la sociedad. El principio organizador de la asimetría de género se basa en la relación de dominación que los hombres tienen sobre las mujeres (Daune-Richard \& Hurtig, 1995). Esta relación de poder existente entre las dos categorías de género se expresa tanto a nivel social como individual.

Diversas investigaciones han señalado que en el sistema androcéntrico los hombres juegan el rol de referentes. Según Daune-Richard y Hurtig (1995), las asimetrías que se dan como resultado de la asimilación androcéntrica forman el núcleo duro del sistema de género. En la representación de los sexos, el sexo femenino se define en base al sexo masculino, sin embargo, el sexo masculino no se define en base al sexo femenino. La división entre masculino y femenino estructura el conjunto de la vida social, las prácticas y las representaciones. Y es el principio masculino el que prevalece sobre el femenino (Bourdieu, 1998). Del mismo modo, Amâncio (1997), con su propuesta conceptual de asimetría simbólica, sostiene que lo masculino tiene un valor genérico y como resultado juega el rol de referente ante lo femenino, subrayando así la especificidad de este último.

En el campo de las RS varias investigaciones han evidenciado la organización en base al género de diferentes objetos sociales. Poeschl (2001), por ejemplo, en su estudio sobre la representación social de la inteligencia, identifica elementos específicos y diferenciadores entre la inteligencia femenina y la masculina. Los análisis muestran que las características diferenciales de la inteligencia femenina son el encanto — característica de poco prestigio 
social—, mientras que la inteligencia masculina se caracteriza por el éxito social —característica valorada socialmente. Esta diferencia, según la autora, responde a la posición asimétrica de los hombres y las mujeres en la estructura social. De forma similar, Stewart (2004) encontró que en un enfermero es más característico ser competente, mientras que en una enfermera es ser atenta. Esta activación diferencial permitiría mantener el estatus positivo de los hombres en un ámbito femenino.

\section{El Enfoque Estructural de las Representaciones Sociales}

Las RS constituyen conocimientos prácticos y sociales con el propósito de aprehender, interpretar y reconstruir la realidad (Elejabarrieta, 1991). El estudio de las RS permite explicar fenómenos que, a su vez, son psicológicos y sociales y, en palabras de Moscovici (1984), pone en marcha la denominada mirada psicosocial. La teoría de las RS supone una nueva perspectiva psicosociológica en el conocimiento de los fenómenos sociales, en su naturaleza, origen y en el análisis de las consecuencias que tienen en las personas, grupos y en el contexto social (Valencia \& Elejabarrieta, 2007).

Una de las diferentes versiones de la propuesta de Moscovici (1984), la perspectiva de la Escuela de Aix concretamente, analiza los procesos cognitivos y profundiza en la relación entre representación social y comportamientos. La teoría del núcleo central se basa en la interdependencia de los componentes de las RS (Guimelli, 1995). Al analizar la representación social de un objeto, es necesario tener en cuenta, además del contenido, su organización y estructura. Banchs (2000) subraya el esfuerzo específico realizado desde esta perspectiva en el desarrollo de metodologías que identifiquen la estructura y sus funciones.

El origen de la teoría del núcleo central se encuentra en los trabajos de Abric $(1976,1987)$. En una representación social se pueden distinguir dos tipos de componentes, los elementos del núcleo central y los de la periferia. El núcleo central es el elemento fundamental de la representación, es su componente más estable y el que delimita su organización y significado. Los elementos periféricos se organizan alrededor del núcleo central y en relación con él. Estos elementos se caracterizan por una mayor flexibilidad y son más sensibles al contexto.

El análisis del sistema central permite estudios comparativos, es decir, permite diferenciar la existencia de dos objetos sociales o señalar, por el contrario, la existencia de una única representación con diferentes variantes. Por su lado, es mediante el análisis del sistema periférico que pueden detectarse trasformaciones o cambios en curso.

Con el objetivo de analizar la organización en base al género de la representación social del desempleo, el presente estudio pretendió determinar su contenido y estructura. Formulamos la hipótesis de que el sistema androcéntrico determina y organiza la representación social del desempleo. Es decir, el núcleo de la representación es el mismo tanto en el desempleo masculino como femenino y las diferencias se gestionan en el sistema periférico. Finalmente, analizamos y comparamos las respuestas dadas por hombres y mujeres para analizar la influencia del posicionamiento de género.

\section{Método}

\section{Participantes}

Participaron en el estudio 231 estudiantes de formación profesional del Departamento de los Pirineos Atlánticos, en la región de Aquitania en Francia. Los jóvenes (102 mujeres y 129 hombres; edad media 21 años) cursaban el segundo curso del ciclo profesional (el último, después del cual la mayoría se orienta al mercado laboral) de tres centros de formación tecnológica elegidos en forma intencionada. De cada centro elegimos también en forma intencionada los 
cursos cuyo itinerario formativo y rama profesional presentaban una presencia equilibrada de mujeres y hombres.

\section{Instrumento y Análisis}

El instrumento que utilizamos fue un cuestionario compuesto de preguntas abiertas (de evocación) y preguntas cerradas (de refutación). Con el fin de simplificar y reducir la extensión del mismo, utilizamos dos tipos: uno con preguntas sobre el desempleo en general y el desempleo masculino (cuestionario masculino) y otro sobre el desempleo en general y el desempleo femenino (cuestionario femenino).

Por medio de las preguntas de evocación se tiene acceso al contenido de la representación (Vergès, 1992). En cada cuestionario utilizamos dos preguntas de evocación, una sobre el desempleo en general y una sobre una mujer o un hombre en desempleo (según el cuestionario femenino o masculino). En el caso, por ejemplo, de la pregunta sobre una mujer en desempleo, planteamos la siguiente pregunta: "¿Qué palabras o expresiones te vienen a la cabeza al pensar en una mujer en desempleo? (escribe de 4 a 8 respuestas)".

Codificamos las respuestas en base al orden de respuesta (primera respuesta $=1$; segun$\mathrm{da}=2$; etc.). En base a las respuestas obtuvimos los elementos susceptibles de pertenecer al núcleo central y definimos las diferentes periferias. Para ello, tuvimos en cuenta la frecuencia y el rango medio de las palabras evocadas. La frecuencia intermedia se establece para crear una división entre las palabras muy frecuentes y las menos frecuentes. Como punto de referencia tomamos el promedio de frecuencias de todas las palabras evocadas con una frecuencia mínima. El rango medio se establece para crear una división entre las palabras prioritarias y secundarias. La frecuencia mínima se establece para eliminar del análisis aquellas palabras cuya frecuencia es muy pequeña o residual. A menudo se establece una frecuencia mínima de cinco (Vergès, 2003). Una vez establecidos los puntos de corte, obtuvimos la información clasificada en cuatro cuadrantes. Los elementos susceptibles de pertenecer al núcleo central los encontramos en el primer cuadrante (alta frecuencia y citados en primer lugar). En el último cuadrante (con palabras poco frecuentes y citadas en segundo lugar) encontramos los elementos más periféricos de la representación o segunda periferia. En el segundo y tercer cuadrantes aparecen los elementos periféricos o primera periferia.

Junto con el análisis lexicográfico, realizamos una categorización con todas las palabras para estudiar las relaciones entre diversas categorías mediante el Índice de Implicación. Este índice señala el porcentaje en que las personas que hacen referencia a algún término de una categoría también lo hacen a términos de otra categoría. El análisis lexicográfico y de categorías lo realizamos con los programas Evoc2000 y Simi2003 (Vergès, 2003) y la comparación de las respuestas dadas por los hombres y las mujeres, mediante el programa Complex (Bonnec, Roussiau \& Vergès, 2002; Vergès 2003).

Para determinar el núcleo central utilizamos cuestionarios de refutación por inducción de escenario ambiguo, es decir, sin delimitar claramente a qué estatus se hacía referencia (Moliner, 1994). Quisimos verificar la centralidad de tres ítems: búsqueda de empleo, problemas financieros y exclusión social. La centralidad de estos ítems había sido señalada en estudios previos dirigidos por Flament (Douroudi, Gialis \& Terracciano, 1994; Hernández \& Marty, 1995; Jannoyer \& Leclerc, 1994), así como por el estudio previo realizado por Valencia y Larrañaga (2004). El cuestionario de refutación se presenta en forma de doble negación, es decir, con el 
enunciado en negativo. El escenario que propusimos en el caso de una mujer desempleada fue el siguiente: "La Señora X no tiene una actividad remunerada, no tiene obligaciones horarias y no busca un empleo. ¿Piensas que la Señora X es ...?” Se proponen seis estatus y se solicitó que señalasen con una $\mathrm{X}$ la respuesta más adecuada: no, probablemente no, probablemente sí y sí. Los seis estatus propuestos fueron los siguientes: minusválida, mujer desempleada, mujer sola con hijos/as a su cargo, desempleada de más de 50 años, rentista, jubilada y ama de casa (ídem para el caso masculino). Si la mayoría de respuestas (más del 70\%) se concentra en las respuestas no y probablemente no, se deduce que el ítem, en este caso búsqueda de empleo, corresponde a dicho estatus (Vergès, 2001).

Finalmente, para determinar si los hombres y las mujeres del estudio compartían la misma representación del desempleo, comparamos, por medio del test Kolmogorov-Smirnov, los promedios de las respuestas de los cuestionarios de refutación y, por medio de $t$ de Student, el léxico utilizado en las preguntas de evocación.

\section{Procedimiento}

El cuestionario fue autoaplicado por el alumnado, después de una breve presentación del equipo investigador, en la que se le informó de nuestro interés en conocer su opinión en relación al desempleo y se le pidió su colaboración voluntaria. La mayoría del alumnado dio su consentimiento verbal, si bien algunas personas rehusaron participar en el estudio y dejaron el cuestionario en blanco. Los datos los recogimos en febrero del 2008. De forma aleatoria y en horario lectivo en el aula, a la mitad le asignamos el cuestionario masculino (46 mujeres y 62 hombres) y al resto, el cuestionario femenino (56 mujeres y 67 hombres).

\section{Resultados}

Primeramente describimos los resultados obtenidos en las tres preguntas de evocación y luego presentamos los resultados de los dos cuestionarios de refutación. Finalmente, presentamos un análisis comparativo entre las respuestas dadas por los hombres y las mujeres.

\section{Las Preguntas de Evocación}

En la pregunta sobre el desempleo en general analizamos el 68,4\% de las evocaciones (palabras que habían sido evocadas al menos cinco veces) y los puntos de corte fueron: frecuencia intermedia 27 y rango medio 3,1. Las palabras susceptibles de pertenecer al núcleo central se encuentran en el primer cuadrante de la Tabla 1. Estas palabras son: empleo, trabajo, buscar, dificultades, problemas, dinero, financieros y precariedad. Junto con estas palabras encontramos otras que indican carencia de empleo: falta y no. La representación social del desempleo incorpora como aspectos centrales las consecuencias económicas (problemas financieros, falta de dinero) y se define en relación al empleo (estar sin empleo y en busca de empleo). En la periferia $\left(2^{\circ}\right.$ y $3^{\text {er }}$ cuadrantes), junto con elementos que hacen referencia a las dimensiones centrales (inactivo, inactividad, renta, salario, pobreza, miseria, aprietos), aparecieron términos que hacen referencia a las ayudas económicas (RMI — renta mínima de inserción-), las estructuras de apoyo (ANPE - Agencia Nacional para el Empleo- y ASSEDIC - Asociación para el Empleo en la Industria y el Comercio- que gestiona las ayudas), la amenaza de exclusión social (exclusión, social), la vulnerabilidad específica ante la que se encuentra la juventud (jóvenes) y las consecuencias psicológicas que puede conllevar (depresión). 
Tabla 1

Frecuencia de Evocaciones Sobre el Desempleo en General

\begin{tabular}{|c|c|c|c|}
\hline Frecuencia $\geq 27$ & Rango medio $<3,1$ & Frecuencia $\geq 27$ & Rango medio $\geq 3,1$ \\
\hline Dinero & 56 & ANPE & 54 \\
\hline Dificultades & 70 & RMI & 31 \\
\hline Empleo & 68 & Exclusión & 27 \\
\hline Financieros & 30 & Social & 27 \\
\hline Falta & 37 & & \\
\hline No & 77 & & \\
\hline Problemas & 40 & & \\
\hline Precariedad & 43 & & \\
\hline Buscar & 64 & & \\
\hline Trabajo & 68 & & \\
\hline Frecuencia $<27$ & Rango medio $<3,1$ & Frecuencia $<27$ & Rango medio $\geq 3,1$ \\
\hline ASSEDIC & 8 & Ayudas & 7 \\
\hline Depresión & 24 & Subsidio & 19 \\
\hline Aprietos & 23 & Desempleo & 14 \\
\hline Infierno & 20 & Familia & 13 \\
\hline Inactivo & 7 & Vago & 7 \\
\hline Inactividad & 11 & Formación & 8 \\
\hline Jóvenes & 24 & Libre & 10 \\
\hline Miseria & 16 & Despido & 9 \\
\hline Pobreza & 17 & Motivación & 10 \\
\hline Personas & 9 & Perder & 17 \\
\hline Renta & 16 & Más & 11 \\
\hline Salario & 13 & Rechazo & 8 \\
\hline Sin & 18 & Remuneración & 8 \\
\hline \multirow[t]{7}{*}{ Demasiado } & 8 & Sociedad & 8 \\
\hline & & Estrés & 11 \\
\hline & & Tiempo & 14 \\
\hline & & Tristeza & 7 \\
\hline & & Encontrar & 12 \\
\hline & & Vida & 16 \\
\hline & & Economía & 8 \\
\hline
\end{tabular}

Nota. Se visualizan las palabras con una frecuencia mínima mayor a 5.

En el desempleo femenino analizamos el 59\% de las evocaciones. Los puntos de corte fueron: frecuencia intermedia 15 y rango medio 2,6 (ver Tabla 2). Las palabras susceptibles de pertenecer al núcleo central son: dificultades, problemas, discriminación, hijos/as, ama de casa, empleo y trabajo. Encontramos, a su vez, otros elementos unidos a algunas de estas palabras, indicando bien su carencia (no tener trabajo, no tener dinero), bien su magnitud (más dificultades). Las palabras hombre y mujer, por su parte, reflejan el efecto comparador que se activa ante la pregunta sobre una mujer en desempleo (lo mismo que un hombre en desempleo, las mujeres tienen más dificultades que los hombres). La representación, en el caso femenino, supone los mismos elementos centrales del desempleo en general: los aspectos económicos y la dimensión laboral. El desempleo femenino recogió también el aspecto socio-estructural discriminatorio que sufren las mujeres (discriminación y sexismo en la periferia), pero incorporó, al 
mismo tiempo, un paralelismo entre el hecho de estar desempleada y ser un ama de casa (ama de casa e hijos/as). En el mismo sentido, en la periferia apareció la dimensión familiar (familia, limpieza del hogar y crianza), cuyo contexto de evocación recoge esta dualidad. En la periferia ( $2^{\circ}$ y $3^{\text {er }}$ cuadrantes) encontramos también términos que hacen referencia a algunas consecuencias psicológicas (estrés) y sociales (exclusión).

Tabla 2

Frecuencia de Evocaciones Sobre una Mujer en Desempleo

\begin{tabular}{lclc}
\hline \multicolumn{1}{c}{ Frecuencia $\geq 15$} & Rango medio $<2,6$ & Frecuencia $\geq 15$ & Rango medio $\geq 2,6$ \\
\hline Dificultades & 43 & Familia & 22 \\
Discriminación & 24 & & \\
Empleo & 22 & & \\
Hijos/as & 45 & \\
Mujer & 17 & \\
Ama de casa & 25 & \\
Hombre & 21 & \\
No & 15 & \\
Más & 26 & \\
Problemas & 20 & \\
Trabajo & 20 & \\
\hline
\end{tabular}

\begin{tabular}{lclc}
\hline \multicolumn{1}{c}{ Frecuencia $<15$} & Rango medio $<2,6$ & Frecuencia $<15$ & Rango medio $\geq 2,6$ \\
\hline Dinero & 12 & Necesidades & 6 \\
Desempleo & 6 & Depresión & 7 \\
Exclusión & 7 & Financieros & 12 \\
Limpieza del hogar & 7 & Desigualdad & 10 \\
Perder & 6 & Falta & 12 \\
Buscar & 11 & Motivación & 6 \\
Salario & 6 & Precariedad & 12 \\
Sexismo & 6 & Social & 12 \\
Estrés & 7 & Vida & 6 \\
Encontrar & 10 & & \\
Crianza & 9 & & \\
\hline
\end{tabular}

Nota. Se visualizan las palabras con una frecuencia mínima mayor a 5.

En el desempleo masculino analizamos el 49,1\% de las evocaciones. Los puntos de corte que establecimos fueron: frecuencia intermedia 12 y rango medio 2,8 (ver Tabla 3). Las palabras susceptibles de pertenecer al núcleo central son: dificultades, problemas, empleo, trabajo y familia. Junto con estas palabras encontramos la carencia de algunos de estos aspectos con la palabra no. La representación en el caso masculino supone los elementos centrales del desempleo en general: los aspectos económicos y la dimensión laboral. Además, visibilizó el rol tradicional de los hombres como cabezas de familia (proveer a la familia, familia que alimentar). En la periferia ( $2^{\circ}$ y $3^{\text {er }}$ cuadrantes) encontramos la estructura de soporte (ANPE), las ayudas económicas (RMI), algunas consecuencias psicológicas (depresión, confianza) y sociales (exclusión), junto con la estigmatización hacia el desempleado (vago), derivada de la asunción de falta de motivación a la hora de buscar empleo. 
Tabla 3

Frecuencia de Evocaciones Sobre un Hombre en Desempleo

\begin{tabular}{lclc}
\hline \multicolumn{1}{c}{ Frecuencia $\geq 12$} & Rango medio $<2,8$ & Frecuencia $\geq 12$ & Rango medio $\geq 2,8$ \\
\hline Dificultades & 22 & Falta & 15 \\
Empleo & 19 & Motivación & 13 \\
Familia & 25 & Buscar & 19 \\
No & 26 & & \\
Problemas & 18 & & \\
Trabajo & 14 & & \\
\hline \multicolumn{1}{c}{ Frecuencia $<12$} & Rango medio $<2,8$ & Frecuencia $<12$ & Rango medio $\geq 2,8$ \\
\hline ANPE & 9 & Dinero & 10 \\
RMI & 6 & Diplomas & 9 \\
Confianza & 7 & Hombre & 8 \\
Depresión & 9 & Vergüenza & 8 \\
Exclusión & 9 & Perder & 10 \\
Vago & 9 & Precariedad & 7 \\
Esposa & 6 & Estrés & 6 \\
Financieros & 9 & Vida & 6 \\
Más & 7 & & \\
Sin & 11 & & \\
Social & 6 & & \\
Encontrar & 7 & & \\
\hline
\end{tabular}

Nota. Se visualizan las palabras con una frecuencia mínima mayor a 5.

En relación al análisis de categorías, clasificamos las evocaciones en 13 categorías: (1) Relación al trabajo (e.g., trabajo, desempleo), (2) Formas de hacer frente (e.g., buscar, entrevistas), (3) Dificultades (e.g., problemas, necesidades), (4) Aspectos económicos (e.g., dinero, gastos), (5) Privación (e.g., sin, falta), (6) Prestaciones (e.g., ayudas, RMI), (7) Familia (e.g., hijos/as, pareja), (8) Formación (e.g., estudiar, cursillos), (9) Aspectos sociales (e.g., exclusión, igualdad), (10) Aspectos individuales (e.g., angustia, desmotivación), (11) Categorías sociales (e.g., mujeres, jóvenes), (12) Sistema y estructura (e.g., gobierno, capitalismo) y (13) Dimensión descriptiva (e.g., catástrofe, larga duración).

En la pregunta sobre el desempleo en general las categorías centrales, las más compartidas y citadas en primer lugar, fueron tres: Relación al trabajo (1), utilizada por el 62,8\% de la muestra y rango medio 2,8; Dificultades (3), utilizada por el 56,7\% y rango medio 2,7; y Aspectos económicos (4), utilizada por el $64,1 \%$ y rango medio 2,9 .

Los análisis de similitud que realizamos para visualizar la inclusión de campos semánticos (ver Figura 1) mostraron que la mayoría de las categorías presentaba altos índices de implicación (más del 60\%) en relación a dos categorías centrales, los Aspectos económicos (4) y su Relación al trabajo (1). En torno a estas dos dimensiones se dividen dos discursos. Por un lado, al ámbito laboral (1) se vincularon estrechamente las categorías sociales (11), los aspectos descriptivos del desempleo (13) y los aspectos estructurales (12). Por otro lado, la dimensión económica (4) se vinculó estrechamente con las dificultades de estar en desempleo (3) y los aspectos sociales (9). 
Finalmente, cabe señalar que la dimensión que recoge las dificultades de estar en desempleo (3) se presentó en un segundo nivel, con altos índices de implicación por parte de los aspectos individuales (10), sociales (9), familiares (7) y estructurales (12).

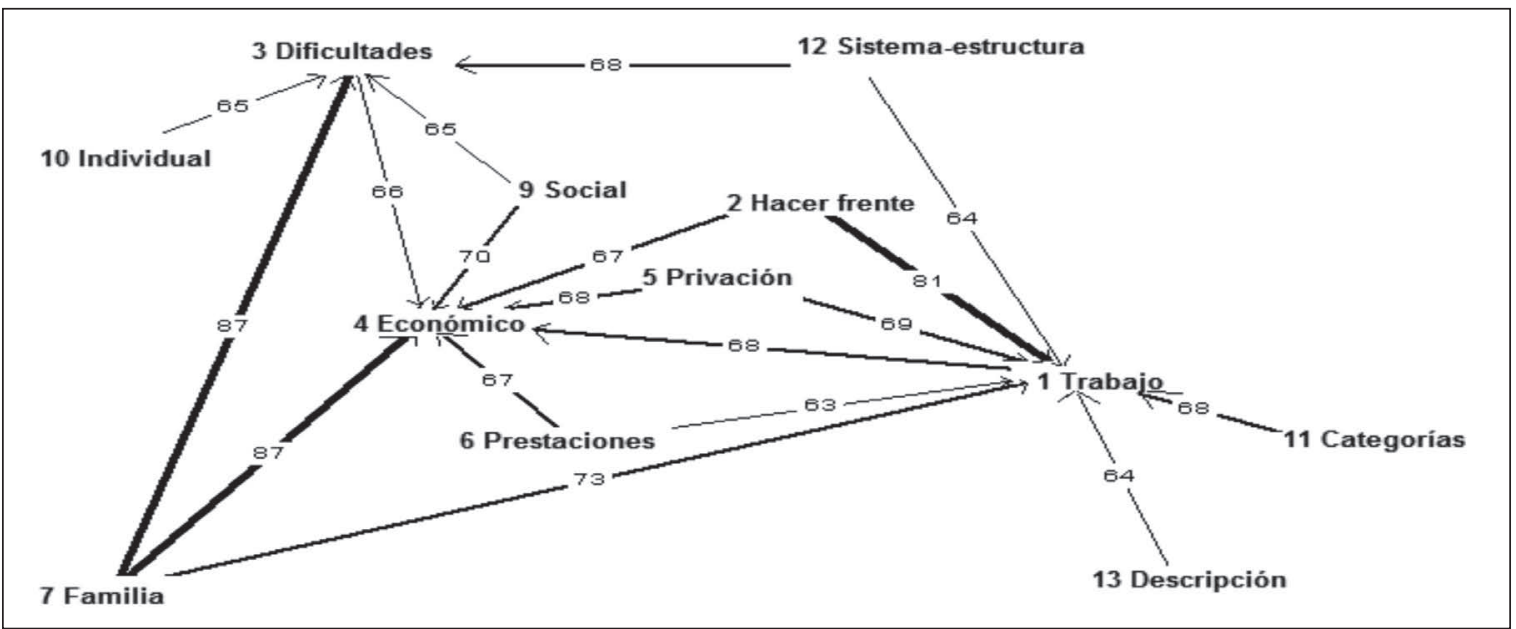

Figura 1. Índice de implicación entre las categorías sobre el desempleo en general (\%).

Las categorías centrales en el caso del desempleo femenino fueron tres (ver Figura 2): Relación al trabajo (1), utilizada por un 48\% y rango medio 2,4; Familia (7), utilizada por un $69,1 \%$ y rango medio 2,4; y Aspectos sociales (9), utilizada por un 52,8\% y rango medio 2,4.

El análisis de similitud mostró que todas las dimensiones presentaban altos índices de implicación (más del 60\%) con la dimensión familiar (7). Las dificultades (3) y la dimensión laboral (1) estaban en un segundo nivel. Mientras que las prestaciones (6) se vinculaban estrechamente con la dimensión laboral (1), los aspectos económicos (4) se asociaban con las dificultades (3).

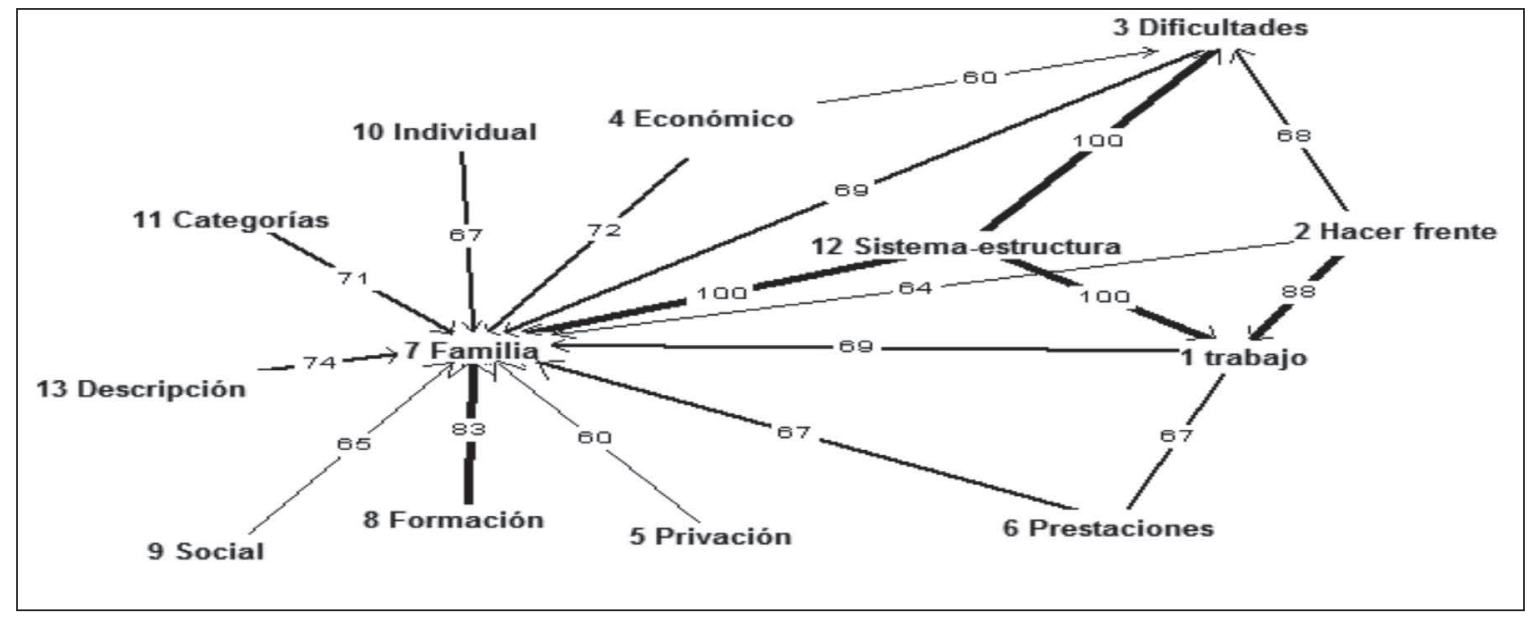

Figura 2. Índice de implicación entre las categorías sobre el desempleo femenino (\%). 
Las categorías centrales en el caso del desempleo masculino fueron cuatro (ver Figura 3): Relación al trabajo (1), utilizada por un 48,6\% y rango medio 2,6; Dificultades (3), utilizada por un $46 \%$ y rango medio 2,6; Privación (5), utilizada por un 42,1\% y rango medio 2,7; y Aspectos individuales (10), utilizada por un $66,4 \%$ y rango medio 2,9 .

El análisis de similitud mostró que la mayoría de las categorías presentaba altos índices de implicación con la dimensión individual (10). Las dimensiones laboral (1) y de dificultades (3) se presentaban en un segundo nivel. Mientras la familia (7) y los aspectos estructurales (12) se vincularon a la dimensión de dificultades (3), fueron las prestaciones (6), las formas de afrontar (2) y los aspectos económicos (4) las que se vincularon a la dimensión laboral (1).

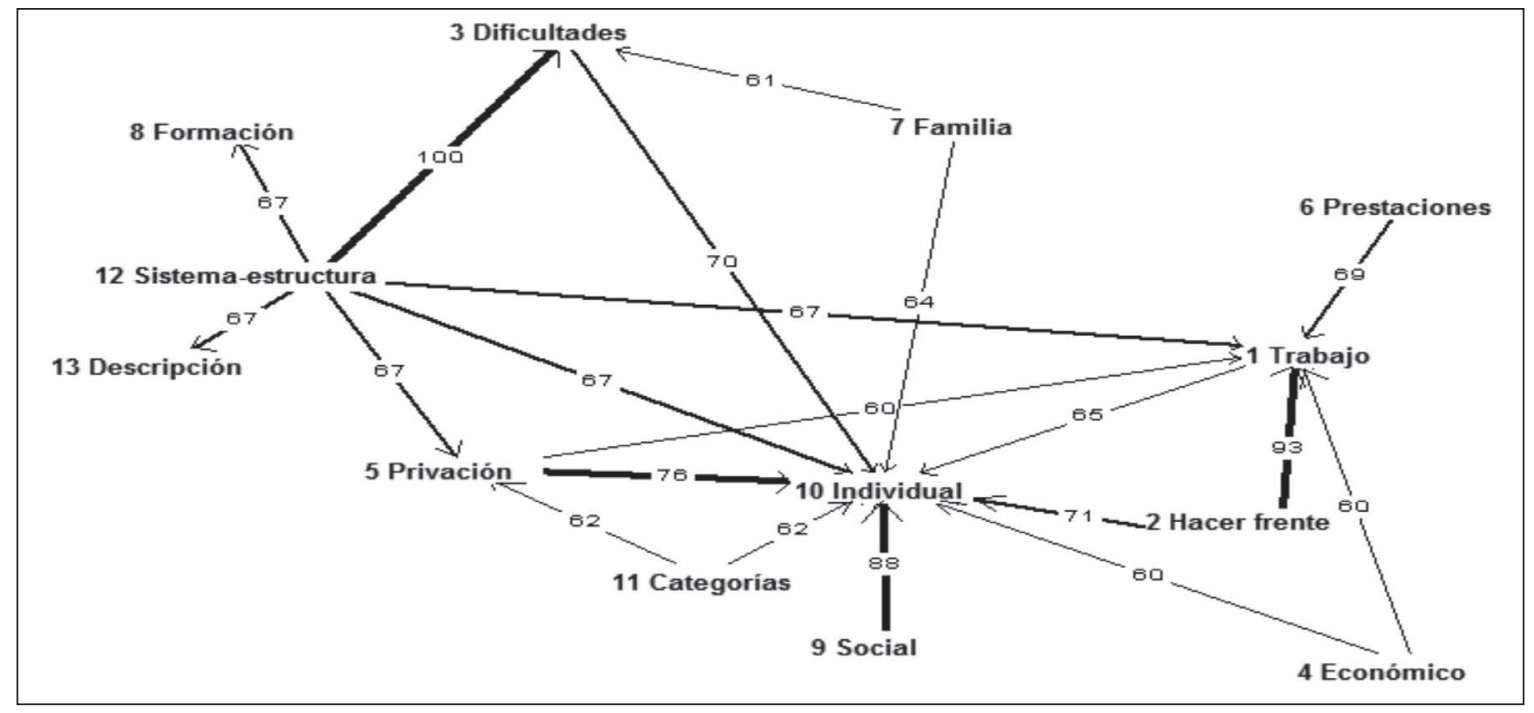

Figura 3. Índice de implicación entre las categorías sobre el desempleo masculino (\%).

\section{Los Cuestionarios de Refutación}

En el cuestionario femenino de refutación (ver Tabla 4) los análisis mostraron que el ítem problemas financieros se encontraba íntimamente unido a los estatus de minusválida, desempleada y mujer sola con hijos/as a cargo. El ítem exclusión social se asoció con minusválida y desempleada y el ítem búsqueda de empleo, con el estatus de desempleada. Es el estatus de desempleada el que recogió los tres ítems como centrales. Por otro lado, los estatus que más se alejaban eran ama de casa y rentista. 
Tabla 4

Resultados Principales del Cuestionario de Refutación Sobre una Mujer en Desempleo (\%)

\begin{tabular}{lcccccc}
\hline \multicolumn{1}{c}{ Ítems x estatus } & Sí & $\begin{array}{c}\text { Probable- } \\
\text { mente Sí }\end{array}$ & Total Sí & $\begin{array}{c}\text { Probable- } \\
\text { Mente No }\end{array}$ & No & Total No \\
\hline Exclu Minusválida & 0,0 & 8,3 & 8,3 & 37,6 & 54,1 & $\mathbf{9 1 , 7}$ \\
PbFi Mujer con hijos/as a cargo & 3,0 & 13,5 & 16,5 & 39,1 & 44,4 & $\mathbf{8 3 , 5}$ \\
Exclu Desempleada & 3,8 & 16,5 & 20,3 & 48,9 & 30,8 & $\mathbf{7 9 , 7}$ \\
Busq Desempleada & 6,8 & 16,5 & 23,3 & 41,4 & 35,3 & $\mathbf{7 6 , 7}$ \\
PbFi Desempleada & 3,8 & 21,8 & 25,6 & 45,1 & 29,3 & $\mathbf{7 4 , 4}$ \\
PbFi Minusválida & 6,0 & 21,8 & 27,8 & 46,6 & 25,6 & $\mathbf{7 2 , 2}$ \\
Exclu Rentista & 35,3 & 39,8 & $\mathbf{7 5 , 1}$ & 16,5 & 8,3 & 24,8 \\
Busq Rentista & 42,1 & 38,3 & $\mathbf{8 0 , 4}$ & 12,0 & 7,5 & 19,5 \\
PbFi Ama de casa & 42,1 & 42,9 & $\mathbf{8 5 , 0}$ & 10,5 & 4,5 & 15,0 \\
Busq Rentista & 51,9 & 29,3 & $\mathbf{8 1 , 2}$ & 14,3 & 4,5 & 18,8 \\
Exclu Ama de casa & 42,1 & 48,1 & $\mathbf{9 0 , 2}$ & 7,5 & 2,3 & 9,8 \\
Busq Ama de casa & 46,6 & 39,1 & $\mathbf{8 5 , 7}$ & 12,0 & 2,3 & 14,3 \\
\hline
\end{tabular}

Nota. Se cruzan los ítems Problemas financieros (PbFi), Exclusión social (Exclu) y Búsqueda de empleo (Busq) con los seis estatus propuestos en cursiva. En negrita se presentan las sumatorias de las columnas afirmativas y negativas que superan el $70 \%$ de las respuestas dadas.

Los análisis del cuestionario masculino de refutación (ver Tabla 5) mostraron que el ítem problemas financieros estaba íntimamente asociado a los estatus de desempleado, hombre solo con hijos/as a su cargo y desempleado de más de 50 años. El ítem exclusión social se asoció con los estatus de minusválido y desempleado y, finalmente, el ítem búsqueda de empleo, con los estatus de desempleado y hombre solo con hijos/as a cargo. En el caso masculino también es el estatus de desempleado el que recogió los tres ítems como centrales. Los estatus que más se alejaban eran rentista y amo de casa. 
Tabla 5

Resultados Principales del Cuestionario de Refutación Sobre un Hombre en Desempleo (\%)

\begin{tabular}{lcccccc}
\hline \multicolumn{1}{c}{ Ítems x Estatus } & Sí & $\begin{array}{c}\text { Probable- } \\
\text { mente Sí }\end{array}$ & Total Sí & $\begin{array}{c}\text { Probable- } \\
\text { mente No }\end{array}$ & No & Total No \\
\hline PbFi Hombre con hijos/as a cargo & 0,0 & 5,9 & 5,9 & 45,4 & 48,7 & $\mathbf{9 4 , 1}$ \\
Exclu Minusválido & 4,2 & 11,8 & 16,0 & 37,8 & 46,2 & $\mathbf{8 4 , 0}$ \\
Busq Desempleado & 5,0 & 14,3 & 19,3 & 41,2 & 39,5 & $\mathbf{8 0 , 7}$ \\
PbFi Desempleado & 3,4 & 16,9 & 20,3 & 45,8 & 33,9 & $\mathbf{7 9 , 7}$ \\
Busq Hombre con hijos/as a cargo & 3,4 & 23,5 & 26,9 & 36,1 & 37,0 & $\mathbf{7 3 , 1}$ \\
PbFi Desempleado+50 & 3,4 & 24,4 & 27,8 & 41,2 & 31,1 & $\mathbf{7 2 , 3}$ \\
Exclu Desempleado & 4,2 & 21,0 & 25,2 & 49,6 & 25,2 & $\mathbf{7 4 , 8}$ \\
Exclu Amo de casa & 18,5 & 54,6 & $\mathbf{7 3 , 1}$ & 18,5 & 8,4 & 26,9 \\
Busq Amo de casa & 26,9 & 52,1 & $\mathbf{7 9 , 0}$ & 13,4 & 7,6 & 21,0 \\
Exclu Rentista & 52,1 & 31,9 & $\mathbf{8 4 , 0}$ & 12,6 & 3,4 & 16,0 \\
Busq Rentista & 61,3 & 26,9 & $\mathbf{8 8 , 2}$ & 9,2 & 2,5 & 11,7 \\
PbFi Rentista & 73,9 & 20,2 & $\mathbf{9 4 , 1}$ & 5,9 & 0,0 & 5,9 \\
\hline
\end{tabular}

Nota. Se cruzan los ítems Problemas financieros (PbFi), Exclusión social (Exclu) y Búsqueda de empleo (Busq) con los seis estatus propuestos en cursiva. En negrita se presentan las sumatorias de las columnas afirmativas y negativas que superan el $70 \%$ de las respuestas dadas.

Los cuestionarios de refutación indicaron que los tres ítems, problemas económicos, exclusión social y búsqueda de empleo, son elementos centrales tanto en el caso femenino como masculino. Los estatus que más se alejan son ama de casa en el caso femenino y rentista en el caso masculino. El test de Kolmogorov-Smirnov indicó diferencias significativas entre las dos distribuciones. En concreto, el estatus de rentista se alejó significativamente más en el caso masculino de los ítems problemas financieros, $Z_{\mathrm{KS}}(231)=2,52, p<0,001$, y exclusión social, $Z_{\mathrm{KS}}(231)=1,33, p=0,059$. El estatus de ama de casa, por su lado, se alejó significativamente más en el caso femenino de los ítems problemas financieros, $Z_{\mathrm{KS}}(231)=2,21, p<0,001$, exclusión social, $Z_{\mathrm{KS}}(231)=1,87, p=0,002$, y búsqueda de empleo, $Z_{\mathrm{KS}}(231)=1,56, p=0,015$.

\section{Comparación de las Repuestas Dadas por los Hombres y las Mujeres}

Las respuestas dadas por los hombres y las mujeres fueron similares, tanto en las preguntas de evocación como en los cuestionarios de refutación. Subrayando esta similitud, pasamos a exponer diferencias detectadas en los análisis comparativos.

Ante la pregunta sobre el desempleo en general, los análisis que realizamos mostraron que las mujeres citaron más que los hombres los términos jóvenes (18 y 6 , respectivamente), $t(1707)$ $=3,02, p<0,1$, pérdida (11 y 6 , respectivamente), $t(1707)=1,69, p<0,1$, y precariedad $(25 \mathrm{y}$ 18 , respectivamente), $t(1707)=1,83, p<0,1$, mientras que los hombres citaron más que las mujeres el término aprietos (18 y 5 , respectivamente), $t(1707)=2,15, p<0,1$. Ante la pregunta sobre el desempleo femenino, las mujeres citaron más la palabra más que los hombres (16 y 10 , respectivamente), $t(887)=1,63, p<0,1$. En las evocaciones sobre el desempleo masculino, las mujeres citaron más que los hombres las palabras falta $(10$ y 5 , respectivamente), $t(755)=$ $1,86, p<0,1$, y $\sin$ (19 y 7 , respectivamente), $t(755)=3,11, p<0,1$. Es necesario indicar que el programa Complex señala como diferencias a tener en cuenta aquellas cuyo $p$ es igual o menor a 0,1 (Vergès, 2003).

En relación a los cuestionarios de refutación, las respuestas dadas por los hombres y las mujeres fueron similares. Sin embargo, cabe destacar una diferencia encontrada. En el cues- 
tionario masculino de refutación los hombres vincularon más que las mujeres el ítem problemas financieros con el estatus de un hombre en desempleo mayor de 50 años, $Z_{\mathrm{KS}}(108)=1,35$, $p=0,053$, y con el estatus de hombre solo con hijos a cargo, $Z_{\mathrm{KS}}(108)=1,45, p=0,031$. En los cuestionarios de refutación sobre el desempleo femenino el test realizado no mostró ninguna diferencia significativa.

\section{Discusión}

Los resultados muestran la consistencia del núcleo central, apoyando la hipótesis de un mismo núcleo central, tanto para el caso masculino como para el femenino. A su vez, el sistema periférico evidenció las especificidades del desempleo femenino frente al masculino. Estos resultados apoyan los resultados obtenidos en el estudio de Valencia y Larrañaga (2004), en el que ya se vislumbraba la existencia de un único objeto social (el desempleo) junto con las especificidades del desempleo femenino. De esta forma hemos podido aumentar el grado de generalización de los resultados, ya que se han mantenido en el tiempo y en diferentes regiones. La lectura de los mismos permite la comprensión de las dinámicas en las relaciones de género que se ponen en marcha ante el desempleo. En concreto, los datos mostraron una clara asimetría en la concreción del desempleo femenino frente al masculino. En este sentido, la aproximación estructural ha permitido articular la dinámica que se establece entre un mismo núcleo central y su regulación cotidiana, en la que el referente en el mundo laboral sigue siendo masculino y el referente en el ámbito doméstico, femenino, el primero valorado socialmente y el segundo menos. Estos resultados evidencian que, si bien conocer y determinar el núcleo de una representación puede ayudar a delimitar la existencia o no de dos RS, es, sin embargo, el análisis del sistema periférico en relación al núcleo central el que permite entender el funcionamiento de la representación en la cotidianeidad.

En concreto, los cuestionarios de refutación mostraron la centralidad de los mismos elementos en el caso femenino y en el masculino: problemas financieros, exclusión social y búsqueda de empleo. De forma paralela, las tres preguntas de evocación identificaron los mismos elementos susceptibles de pertenecer al núcleo central: los aspectos económicos que conllevan estar en desempleo y su relación al trabajo. Sin embargo, el análisis del sistema periférico mediante las preguntas de evocación mostró diferencias entre el caso masculino y el femenino. Mientras que el desempleo femenino recogió la discriminación existente hacia las mujeres y asemejó los estatus de desempleada y ama de casa, el desempleo masculino se asoció con la figura de cabeza de familia y destacó la presión social vivida por los hombres a nivel personal e identitario, así como su responsabilidad. El análisis categorial de las evocaciones mostró que la dimensión central en el caso femenino era claramente la familiar, mientras que en el caso masculino era la individual. En ambos casos el género y las expectativas asociadas al mismo orientan la concreción de la representación. Sin embargo, se nos plantea la necesidad de profundizar en las implicaciones de los posicionamientos sociales asimétricos con poblaciones que tengan una vivencia más directa con el objeto de estudio.

\section{Conclusiones}

Los resultados de este estudio plantean elementos interesantes sobre las dinámicas de la representación social del desempleo y su concreción en el desempleo masculino y el femenino.

Las preguntas de evocación sobre el desempleo femenino, si bien mostraron los elementos comunes y centrales, también evidenciaron algunos elementos diferenciadores. El análisis del sistema periférico mostró un sistema condicional, dando lugar a dos tipos de desempleadas: el ama de casa, que no padece la situación de desempleo como un hombre, y la desempleada pobre que, substituyendo el rol de cabeza de familia, encuentra más dificultades debido al sexismo existente. Al parecer, los cambios en los roles de género que permiten adaptarse a nuevas realidades sociales se instalan en convivencia con modelos tradicionales que permanecen (Sharim, 
2005). En este sentido, sería interesante un análisis más específico de la relación entre núcleo y periferia que permita identificar los mecanismos cognitivos que se utilizan para justificar la representación. Entre otros, gracias a la metodología desarrollada por Guimelli y Rouquette (1993) y su modelo de Esquemas Cognitivos de Base, podrían identificarse los mecanismos cognitivos subyacentes y dar cuenta de los procesos de cambio o resistencia puestos en marcha. En su estudio sobre los valores que los jóvenes atribuyen al trabajo, Márquez y Friemel (2005) identifican que el énfasis en unos valores más individualistas o más colectivistas responde en gran medida a la pertenencia social de los jóvenes, así como a la activación del objeto de representación (trabajo, empleo o desempleo). Estos resultados sugieren que la representación social del desempleo que elaboran los jóvenes constituye una forma de hacer frente a la situación y responde, a su vez, a la mantención de las diferencias sociales (Giron, 2001).

En el caso del desempleo masculino, toma peso la vivencia individual. El empleo, más que un medio para obtener dinero, se erige en parte central de la identidad masculina (Blanch, 1990). Además, al subrayar el papel del hombre como cabeza de familia, el desempleo masculino adquiere consecuencias más graves, ya que afecta no solo al individuo, sino también a su entorno más próximo. Argumentos similares se utilizan para justificar las diferencias salariales entre hombres y mujeres (Silvera, 1995).

El crecimiento de la tasa de actividad femenina ha supuesto un ajuste a nivel periférico de la representación del desempleo. El núcleo central se mantiene, pero permite, a su vez, la incorporación de nuevas prácticas sociales mediante el sistema periférico. El cambio del sistema androcéntrico se presenta difícil, ya que a nivel ideológico hacen falta generaciones y un largo trabajo de modificación del entorno, de relaciones sociales de género y de prácticas de toda naturaleza (Flament \& Rouquette, 2003). Este sistema es compartido por hombres y mujeres de nuestro estudio, dando cuenta de la integración de las relaciones de dominación por los grupos dominados (Bourdieu, 1998; Tafani \& Bellon, 2001). La representación social del desempleo asegura, así, la mantención de la asimetría de género, sosteniendo los estereotipos sobre los hombres y las mujeres, participando en la justificación del sistema (Jost \& Banaji, 1994) y contribuyendo en la reproducción del orden simbólico establecido (Guichard \& Bidot, 1989; Guichard \& Cassar, 1998; Lorenzi-Cioldi, 1997). De acuerdo con los resultados de Alfonso y Poeschl (2006), podemos decir que en la representación social que los y las jóvenes tienen del desempleo perdura una visión tradicional de la división de los papeles entre hombres y mujeres. En este punto cabría cuestionarse el nivel de implicación de los y las participantes con el objeto de representación. En esta investigación participaron estudiantes de formación profesional, lo que puede resultar una limitación a la hora de hacer extensivos los resultados del estudio a poblaciones con otros perfiles. En su estudio Milland (2002) evidenció que las prácticas significativas ayudan a estructurar mejor la representación social. Además, un mayor nivel de implicación podría también acentuar el posicionamiento social de género. En este sentido, una línea interesante de investigación futura consistiría en relacionar los procesos de identidad social con las dinámicas estructurales de las RS. En la medida en que el trabajo se considera una mediación capital en la construcción de la femineidad y de la masculinidad (Molinier, 2002), ahondar en la representación social que los y las jóvenes elaboran en relación al desempleo en general y al desempleo masculino y femenino en particular constituye una forma de analizar las relaciones sociales de género que se ponen en marcha, tanto en relación con la división del espacio (público-empleo, privado-doméstico) como de la valencia diferencial existente entre la dominancia del principio masculino sobre el femenino (Héritier, 2002). Si bien González y Zarco (2004) concluyen en su trabajo que los cambios a nivel socio-estructural están influyendo a nivel psicosocial y concretamente en relación con las actitudes y comportamientos de las y los jóvenes en relación a la actividad laboral, asemejándolos, nos preguntamos si la mayor dificultad de las mujeres a la hora de encontrar un empleo se debe, en parte, a la influencia de la asimetría de género. En este sentido, consideramos que trabajar en la problemática de las relaciones de género puede ayudar a adecuar las políticas de orientación profesional e inserción laboral. 


\section{Referencias}

Abric, J. C. (1976). Jeux, conflits et représentations sociales [Juegos, conflictos y representaciones sociales] (Tesis de Doctorado no publicada), Faculté de Lettres, Aix-Marseille I, Université de Provence, Aix en Provence, Francia.

Abric, J. C. (1987). Coopération, compétition et représentations sociales [Cooperación, competencia y representaciones sociales]. Cousset, Suiza: DelVal.

Alfonso, R. M. \& Poeschl, G. (2006). Representaciones del impacto de la situación de desempleo en las prácticas familiares. Revista de Psicología Social, 21, 241-258.

Amâncio, L. (1997). The importance of being male: Ideology and context in gender identities. Revue Internationale de Psychologie Sociale, 10(2), 79-94.

Aquilini, G. de H. \& Costa, P. L. (2003). O sobre-desemprego e a inatividade das mulheres na metrópole paulista [E] sobre-desempleo y la inactividad de las mujeres en la metrópoli paulista]. Estudos Avançados, 49, 17-34.

Banchs, M. A. (2000). Aproximaciones procesuales y estructurales al estudio de las representaciones sociales. Papers on Social Representations, 9, 3.1-3.15. Extraído de http://www.psr.jku.at/PSR2000/9_3Banch.pdf

Blanch, J. M. (1990). Del viejo al nuevo paro: un análisis psicológico y social. Barcelona, España: Promociones y Publicaciones Universitarias.

Bonnec, Y., Roussiau, N. \& Vergès, P. (2002). Categorical and prototipical analysis: A study on the quality-process in hospital institutions. Revue Européenne de Psychologie Appliquée, 52, 213-220.

Bourdieu, P. (1977). La production de la croyance [La producción de la creencia]. Actes de la Recherche en Sciences Sociales, 13, 3-43. doi:10.3406/arss.1977.3493

Bourdieu, P. (1998). La domination masculine [La dominación masculina]. Paris, Francia: Editions du Seuil.

Cozzarelli, C., Tagler, M. J. \& Wilkinson, A. V. (2002). Do middle-class students perceive poor women and poor men differently? Sex Roles, 47, 519-529. doi:10.1023/A:1022038200071

Daune-Richard, A. M. \& Hurtig, M. C. (1995). Catégories et représentations de sexe: Un débat loin d’être clos [Categorías y representaciones de sexo: un debate lejos de estar cerrado]. En Ephesia (Ed.), La place des femmes: Les enjeux de l'identité et de l'égalité au regard des sciences sociales [El lugar de las mujeres: la cuestión de identidad y la igualdad desde las ciencias sociales] (pp. 426-438). Paris, Francia: La Découverte.

Douroudi, E., Gialis, M. \& Terracciano, M. (1994). La représentation sociale du travail et du chômage chez les travailleurs et les chômeurs qualifiés [La representación social del trabajo y del desempleo en trabajadores y desempleados calificados] (Tesis de Magíster no publicada), Faculté de Lettres, Aix-Marseille I, Université de Provence, Aix en Provence, Francia.

Duru-Bellat, M. (1990). L'école des filles: Quelle formation pour quels rôles sociaux? [La escuela de chicas: ¿qué formación para qué roles sociales?]. Paris, Francia: L'Harmattan.

Elejabarrieta, F. (1991). Representaciones sociales. En A. Echevarría (Ed.), Psicología social sociocognitiva (pp. 223248). Bilbao, España: Desclée de Brouwer.

Flament, C. \& Rouquette, M. L. (2003). Qu'est-ce qu’une représentation sociale? [¿Qué es una representación social?]. En C. Flament \& M. L. Rouquette (Eds.), Anatomie des idées ordinaires: Comment étudier les représentations sociales [Anatomía de las ideas comunes: cómo estudiar las representaciones sociales] (pp. 11-30). Paris, Francia: Armand Colin.

Giron, C. (2001). La représentation d’un objet économique stressant: Les adolescents face au chômage [La representación de un objeto económico estresante: los adolescentes frente al desempleo]. Psychologie Française, 46, 353-363.

González, V. \& Zarco, J. M. (2004). Semejanzas y diferencias de género en las actitudes hacia las demandas del mercado laboral. Revista de Psicología Social, 19, 231-253.

Guichard, J. \& Bidot, H. (1989). Filières scolaires et représentations professionnelles des lycéens [Sectores escolares y representaciones profesionales de los alumnos de secundaria]. Revue Internationale de Psychologie Sociale, 2(4), 123-145.

Guichard, J. \& Cassar, O. (1998). Social fields, habitus and cognitive schemes: Study streams and categorisation of occupations. Revue Internationale de Psychologie Sociale, 11(1), 123-145.

Guimelli, C. (1995). L'étude des représentations sociales [El estudio de las representaciones sociales]. Psychologie Française, 40, 367-374.

Guimelli, C. \& Rouquette, M. L. (1993). Note sur la formalisation des schèmes étranges dans l'étude des représentations sociales [Nota sobre la formalización de esquemas extraños en el estudio de las representaciones sociales]. Les Cahiers Internationaux de Psychologie Sociale, 19, 43-48.

Héritier, F. (2002). Masculin/féminin II. Dissoudre la hiérarchie [Maculino/femenino II. Disolución de la jerarquía]. Paris, Francia: O. Jacob.

Hernández, A. \& Marty, N. (1995). La représentation sociale du travail et du chômage chez les travailleurs et les chômeurs qualifiés [La representación social del trabajo y del desempleo en trabajadores y desempleados calificados] (Tesis de Magíster no publicada), Faculté de Lettres, Aix-Marseille, Université de Provence, Aix en Provence, Francia.

Jannoyer, C. \& Leclerc. M. (1994). La représentation sociale du travail et du chômage chez les travailleurs et les chômeurs qualifiés [La representación social del trabajo y del desempleo en trabajadores y desempleados calificados] (Tesis de Magíster no publicada), Faculté de Lettres, Aix-Marseille I, Université de Provence, Aix en Provence, Francia.

Jost, J. T. \& Banaji, M. R. (1994). The role of stereotyping in system-justification and the production of false consciousness. British Journal of Social Psychology, 33, 1-27. doi:10.1111/j.2044-8309.1994.tb01008.x 
Lorenzi-Cioldi, F. (1997). Professions au masculin et au féminin: Un moyen terme entre le masculin et le féminin? [Las profesiones de lo masculino y lo femenino: ¿un término medio entre lo masculino y lo femenino?]. Revue Internationale de Psychologie Sociale, 10(2), 135-152.

Márquez, E. \& Friemel, E. (2005). Activation des schemes cognitifs de base et actualisation des valeurs associées au travail [Activación de los esquemas cognitivos de base y actualización de los valores asociados al trabajo]. Papers on Social Representations, 14, 1.1-1.28. Extraído de http://www.psych.lse.ac.uk/psr/PSR2005/14_01Mar.pdf

Milland, L. (2002). Pour une approche de la dynamique du rapport entre représentations sociales du travail et du chômage [Un acercamiento a la dinámica de las relaciones entre representaciones sociales del trabajo y del desempleo]. Revue Internationale de Psychologie Sociale, 15(2), 27-56.

Moliner, P. (1994). Les méthodes de repérage et d’identification du noyau des représentations sociales [Los métodos para recoger e identificar el núcleo de las representaciones sociales]. En C. Guimelli (Ed.), Structures et transformations des représentations sociales [Estructuras y transformaciones de las representaciones sociales] (pp.199-232). Neuchâtel, Suiza: Delachaux et Niestlé.

Molinier, P. (2002). Féminité sociale et construction de l'identité sexuelle: Perspectives théoriques et cliniques en psychodynamique du travail [Femineidad social y construcción de la identidad sexual: perspectivas teóricas y clínicas en la psicodinámica del trabajo]. L’Orientation Scolaire et Professionnelle, 31, 565-580.

Moscovici, S. (1984). The phenomenon of social representations. En R. M. Farr \& S. Moscovici (Eds.), Social representations (pp. 3-69). Cambridge, Reino Unido: Cambridge University Press.

Organización Internacional del Trabajo (2007). Tendencias mundiales del empleo de las mujeres. Resumen marzo de 2007. Genève, Suiza: Autor. Extraído del sitio Web de la OIT: http://www.ilo.org/empelm/what/pubs/lang--es/ docName--WCMS_114356/index.htm

Poeschl, G. (2001). Social comparison and differentiation strategies in social representations of intelligence. Swiss Journal of Psychology, 60, 15-26. doi:10.1024//1421-0185.60.1.15

Sharim, D. (2005). La identidad de género en tiempos de cambio: una aproximación desde los relatos de vida. Psykhe, 14(2), 19-32. doi:10.4067/S0718-22282005000200002

Silvera, R. (1995). Les inégalités de salaires entre hommes et femmes ou comment expliquer ce qui reste "inexplicable" [Las desigualdades de los salarios entre hombres y mujeres o cómo explicar lo "inexplicable”]. En Ephesia (Eds.), La place des femmes: Les enjeux de l'identité et de la l'égalité au regard des sciences sociales [El lugar de las mujeres: la cuestión de identidad e igualdad desde las ciencias sociales] (pp. 546-551). Paris, Francia: La Découverte.

Stewart, I. (2004). La représentation sociale de la profession d'infirmier/ère: Effets de contexte liés à un changement de genre [La representación social de la profesión de enfermero/a: efectos del contexto en relación al cambio de género]. Psychologie et Société, 7, 171-209.

Tafani, E. \& Bellon, S. (2001). Principe d’homologie structurale et dynamique représentationnelle [Principio de homología estructural y dinámica representacional]. En P. Moliner (Ed.), La dynamique des représentations sociales [La dinámica de las representaciones sociales] (pp. 163-194). Grenoble, Francia: Presses Universitaires de Grenoble.

Torns, T. (1998/2000). When exclusion is socially acceptable: The case of Spain. En J. Jenson, L. Laufer \& M. Maruani (Eds.), The gendering of inequalities: Women, men and work (pp. 216-227, H. Arnold, Trad.). Aldershot, Reino Unido: Ashgate.

Valencia, J. F. \& Elejabarrieta, F. J. (2007). Aportes sobre la explicación y el enfoque de las representaciones sociales. En T. Rodríguez \& M. L. García (Coords.), Representaciones sociales. Teoría e investigación (pp. 89-136). Guadalajara, México: Universidad de Guadalajara.

Valencia, J. F. \& Larrañaga, M. (2004). Representaciones sociales y paro femenino: ¿significados compartidos en proceso de transformación? Psicologia: Teoria e Prática, 6(Número especial), 17-30. Extraído de http://pepsic. bvsalud.org/pdf/ptp/v6nspe/v6nspea02.pdf

Vergès, P. (1992). L'évocation de l'argent: Une méthode pour la définition du noyau central d'une représentation [La evocación del dinero: un método para la definición del núcleo central de una representación]. Bulletin de Psychologie, 45, 203-209.

Vergès, P. (2001). L'analyse des représentations sociales par questionnaires [El análisis de las representaciones sociales mediante cuestionarios]. Revue Française de Sociologie, 42, 537-561. Extraído de http://www.persee.fr/ web/revues/home/prescript/article/rfsoc_0035-2969_2001_num_42_3_5373

Vergès, P. (2003). Ensemble de programmes permettant l'analyse des évocations, evoc2000 [Conjunto de programas para el análisis de las evocaciones, evoc2000 ] [CD Rom]. Aix en Provence, Francia: Laboratoire Méditerranéen de Sociologie.

Vouillot, F., Blanchard, S., Marro, C. \& Steinbruckner, M. L. (2004). La division sexuée de l'orientation et du travail: Une question théorique et une question de pratiques [La división sexual de la orientación y del trabajo: una cuestión teórica y una cuestión de prácticas]. Psychologie du Travail et des Organisations, 10, 277-291. doi:10.1016/ j.pto.2004.07.004

Fecha de recepción: Marzo de 2011.

Fecha de aceptación: Marzo de 2012. 